\title{
Prognostic Significance of Neoadjuvant Rectal Score and Indication for Postoperative Adjuvant Therapy in Rectal Cancer Patients After Neoadjuvant Chemoradiotherapy
}

\author{
KIYOSHI MAEDA ${ }^{1,2}$, MASATSUNE SHIBUTANI $^{2}$, AKIKO TACHIMORI $^{1}$, \\ TAKAFUMI NISHII ${ }^{1}$, NAOKI AOMATSU ${ }^{1}$, TATSUNARI FUKUOKA ${ }^{2}$, HISASHI NAGAHARA ${ }^{2}$, \\ HIROSHI OTANI $^{2}$, TORU INOUE ${ }^{1}$ and MASAICHI OHIRA ${ }^{1}$ \\ ${ }^{1}$ Department of Gastroenterological Surgery, Osaka City General Hospital, Osaka, Japan; \\ ${ }^{2}$ Department of Gastroenterological Surgery, Osaka City University Graduate School of Medicine, Osaka, Japan
}

\begin{abstract}
Background/Aim: Neoadjuvant chemoradiotherapy $(C R T)$ is a standard treatment for patients with clinical Stage II/III rectal cancer. However, the benefit of postoperative adjuvant chemotherapy for patients after neoadjuvant CRT is uncertain. Recently, neoadjuvant rectal (NAR) score was suggested as an independent prognostic factor for patients with rectal cancer after neoadjuvant CRT. The aim of this study was to examine the prognostic significance of NAR score in rectal cancer patients who underwent neoadjuvant CRT followed by surgery, and to investigate which patients may benefit from postoperative adjuvant therapy. Patients and Methods: A total of 72 patients who underwent neoadjuvant CRT followed by $R 0$ resection for clinical stage II /III rectal cancer were evaluated. The correlation between NAR score, various clinicopathological factors and disease recurrence were evaluated. Results: Disease recurrence was significantly more often observed in patients with incomplete neoadjuvant $C R T$, tumor regression grade (TRG) 3-4, and high NAR score. Multivariate analysis revealed that NAR score was an independent predictor of disease recurrence. Conclusion: NAR score may be one of the predictive markers for disease recurrence in patients who underwent neoadjuvant CRT followed by surgery for rectal cancer. Patients with a low NAR score may benefit form postoperative adjuvant chemotherapy.
\end{abstract}

This article is freely accessible online.

Correspondence to: Kiyoshi Maeda, MD, Department of Gastroenterological Surgery, Osaka City General Hospital, 2-13-22 Miyakojima-hondori, Miyakojima-ku, Osaka, 534-0021, Japan. Tel: +8169291221, Fax:+8166290886, e-mail: m1378386@med.osakacu.ac.jp

Key Words: Rectal cancer, chemoradiotherapy, neoadjuvant rectal score, adjuvant therapy.
A number of studies have demonstrated that neoadjuvant chemoradiotherapy (CRT) significantly reduces the risk of local recurrence in locally advanced rectal cancer (LARC) (15). The National Comprehensive Cancer Network (NCCN) guidelines (6) recommended neoadjuvant CRT followed by surgery for clinical (c) Stage II or III rectal cancer. The NCCN guidelines also recommend postoperative combined administration of fluoropyrimidine (FL) and oxaliplatin (OX), such as FOLFOX and CapeOX, as adjuvant chemotherapies for patients with cStage II or III disease regardless of the surgical pathology results. However, unfortunately, the role of postoperative adjuvant chemotherapies remains controversial in patients who underwent neoadjuvant CRT followed by R0 resection for rectal cancer (7-14). Bujko et al. (7) conducted a meta-analysis on 4 trials involving 2398 patients who underwent neoadjuvant CRT followed by surgery, and reported that the use of postoperative adjuvant chemotherapy is not based on scientific evidence. Whereas, Petrelli et al. (8) analyzed data from 5 randomized trials and 10 retrospective studies, and reported that adjuvant chemotherapy improved both 5-year overall survival and 5-year disease-free survival.

FOLFOX or CapeOX administration may cause grade III or more severe adverse events (AE) in $30-40 \%$ of the patients who receive these treatments (15). Oxaliplatin-induced neuropathy is one of the most frequently observed $\mathrm{AE}$ and may reduce the quality of life. Under rare circumstances, $\mathrm{AE}$ may be life threatening and may require hospitalization. Owing to these observations, some experts have questioned whether adjuvant chemotherapy should be administered to all patients who undergo neoadjuvant CRT followed by R0 resection.

Therefore, patients with higher risk of disease recurrence and poor survival, who may be candidates for postoperative adjuvant chemotherapy, were selected.

According to the prognosis of the patients with neoadjuvant therapy, the degree of tumor downstaging is thought to be more important than the absolute ypStage. The neoadjuvant 
rectal (NAR) score was proposed by the National Surgical Adjuvant Breast and Bowel Project (NSABP) as a surrogate end point to assess preoperative treatment efficacy in clinical trials of rectal cancer (16). It is calculated based on data only using the clinical $\mathrm{T}$ stage and pathological $\mathrm{T}$ and $\mathrm{N}$ stages and standardizes the degree of tumor downstaging.

The aim of this study was to examine the prognostic significance of NAR score in patients with LARC who underwent neoadjuvant CRT followed by R0 resection, and to investigate which patients may benefit from postoperative adjuvant therapy.

\section{Patients and Methods}

This study was approved by the institutional review board at Osaka City University Hospital and Osaka City General Hospital. Each patient gave his/her written informed consent prior to any study procedure.

Patients. We retrospectively collected and reviewed data on patient characteristics and perioperative data after obtaining approval from the review boards of Osaka City General Hospital and Osaka City University Hospital. Between January 2010 and December 2014, a total of 72 consecutive patients who underwent neoadjuvant CRT followed by R0 resection, for previously untreated cStage II /III rectal cancer at our departments, were evaluated. Clinical stage was confirmed by colonoscopy, magnetic resonance imaging (MRI), and computed tomography of the chest, abdomen, and pelvis. When distant metastasis was suspected, positron emission tomography (PET) was also performed. Clinical and pathological staging were determined according to the American Joint Committee (AJCC) TNM staging system. The location of each tumor was within $10 \mathrm{~cm}$ from the anal verge.

In all cases, the disease was considered to be resectable at the time of entry, with no evidence of distant metastases.

Chemoradiotherapy. Radiotherapy was administered using a 4-field conformal coplanar technique (anteroposterior, posteroanterior, right lateral and left lateral fields) and 6-10 MV photon beams. A total dose of 50.4 Gy was administered as 1.8 Gy fractions, five fractions per week, for 5 weeks and 3 days. Preoperative chemotherapy with concurrent radiotherapy included capecitabine $900 \mathrm{mg} / \mathrm{m}^{2}$ or S-1 40 $\mathrm{mg} / \mathrm{m}^{2}$ twice a day during the entire period of radiotherapy.

Surgery. Within 6-10 weeks after completing chemoradiotherapy, surgery with total mesorectal excision was performed. Postoperative complications were recorded not only during hospital admission, but also during the first 30 days after discharge. The severity of surgical complications was scored using the Clavien-Dindo classification of surgical complications (17).

The physician determined whether postoperative adjuvant chemotherapy was administrated or not, and which chemotherapy regimen was chosen.

Pathological evaluation. A postoperative, pathological evaluation of the surgical specimen was performed. A pathological complete response (pCR) was defined as the complete disappearance of all tumor cells. The pathological tumor regression grade (TRG) was
Table I. Characteristics of 72 patients who underwent neoadjuvant chemoradiotherapy followed by surgery for rectal cancer.

\begin{tabular}{lc}
\hline Gender & \\
Male & 56 \\
Female & 16 \\
Age & \\
$\quad \geq 70$ years & 33 \\
$\quad<70$ years & 39 \\
cStage (Pretreatment) & \\
II & 35 \\
IIIB & 19 \\
IIIC & 18 \\
ypStage & \\
0 & 11 \\
I & 20 \\
II & 30 \\
III & 11 \\
Completion of chemoradiotherapy & \\
Complete & 64 \\
Incomplete & 8 \\
Postoperative complications & \\
(Clavien-Dindo IIIa or more) & \\
Present & 14 \\
Absent \\
Postoperative adjuvant chemotherapy & 58 \\
None & \\
FL & 37 \\
FL+OX & 21 \\
Pathological tumor regression grade & 14 \\
1, & \\
3 N 4 & 42 \\
NAR score & 30 \\
$>16$ & 11 \\
$\leq 16$ & 61 \\
\hline
\end{tabular}

FL: Fluoropyrimidine; OX: oxaliplatin; NAR score: neoadjuvant rectal score.

evaluated using histological regression. TRG-1, pCR, absence of viable cancer cells in the resected specimen, TRG-2, presence of residual cancer cells; TRG-3, fibrosis outgrowing residual cancer cells, TRG-4, residual cancer cells outgrowing fibrosis, TRG-5, absence of response (18).

Variables. The following 8 parameters were evaluated as potential risk factors for disease recurrence: age at the time of surgery $(\geq 70$ or $<70$ years), gender, pretreatment cStage, ypStage, TRG, NAR score, completion of neoadjuvant CRT, and the presence of postoperative adjuvant chemotherapy.

The NAR score was developed on the basis of Valentini's nomograms for overall survival incorporating a weighted combination of the pre-CRT clinical T (cT) stage, post CRT pathologic T (ypT) stage and pathological nodal (ypN) stage, and were calculated using the equation $[5 \mathrm{ypN}-3(\mathrm{cT}-\mathrm{ypT})+12]^{2} \div 9.61$ (17). The NAR score was classified as low $(\mathrm{NAR} \leq 16)$ and high $(\mathrm{NAR}>16)$ according to Valentini et al. (17).

Statistical analysis. Statistical analyses were performed using the JMP 10 software program (SAS Institute Japan, Tokyo, Japan). The Chi-square test was used to compare the data. Survival curves were 
Table II. Results of the univariate and multivariate analyses of the prognostic factors in 72 patients who underwent neoadjuvant chemoradiotherapy followed by surgery for rectal cancer.

\begin{tabular}{|c|c|c|c|c|c|c|}
\hline & \multicolumn{2}{|c|}{ Disease recurrence } & \multirow{2}{*}{$\begin{array}{c}\text { Univariate analysis } \\
\text { p-Value }\end{array}$} & \multicolumn{3}{|c|}{ Multivariate analysis } \\
\hline & Present & Absent & & $\overline{p \text {-Value }}$ & OR & $95 \% \mathrm{CI}$ \\
\hline \multicolumn{7}{|l|}{ Gender } \\
\hline Male & 14 & 42 & 0.504 & & & \\
\hline Female & 6 & 10 & & & & \\
\hline \multicolumn{7}{|l|}{ Age } \\
\hline$\geq 71$ years & 13 & 20 & 0.078 & & & \\
\hline$<71$ years & 7 & 32 & & & & \\
\hline \multicolumn{7}{|c|}{ cStage (Pretreatment) } \\
\hline II & 11 & 24 & 0.682 & & & \\
\hline III & 9 & 28 & & & & \\
\hline \multicolumn{7}{|l|}{ ypStage } \\
\hline $0, \mathrm{I}$ & 31 & 5 & 0.098 & & & \\
\hline II, III & 15 & 26 & & & & \\
\hline \multicolumn{7}{|c|}{ Completion of chemoradiotherapy } \\
\hline Complete & 15 & 49 & 0.033 & 0.07 & 1.76 & $0.87-10.83$ \\
\hline Incomplete & 5 & 3 & & & & \\
\hline \multicolumn{7}{|c|}{$\begin{array}{l}\text { Postoperative complications } \\
\text { (Clavien-Dindo IIIa or more) }\end{array}$} \\
\hline Present & 16 & 42 & 0.796 & & & \\
\hline Absent & 4 & 10 & & & & \\
\hline \multicolumn{7}{|c|}{ Postoperative adjuvant chemotherapy } \\
\hline None & 12 & 25 & 0.519 & & & \\
\hline Present & 8 & 27 & & & & \\
\hline \multicolumn{7}{|c|}{ Pathological tumor regression grade } \\
\hline 1,2 & 7 & 35 & 0.026 & 0.03 & 2.93 & $1.08-8.55$ \\
\hline 3,4 & 13 & 17 & & & & \\
\hline \multicolumn{7}{|l|}{ NAR score } \\
\hline$>16$ & 7 & 4 & 0.011 & 0.02 & 3.72 & $1.22-13.7$ \\
\hline$\leq 16$ & 13 & 48 & & & & \\
\hline
\end{tabular}

NAR score: Neoadjuvant rectal score.

created by the Kaplan-Meier method and analyzed using the logrank test. The Cox proportional hazard model was used for the multivariate analysis to identify the independent prognostic factors.

All variables with a $p$-value of $<0.05$ in the univariate analyses were included in a multivariate logistic regression analysis. A $p$ value of $<0.05$ was considered to be statistically significant.

\section{Results}

Patient characteristics. The clinical characteristics of all 72 patients are summarized in Table I. The median age at surgery was 73 years (range $=41-80$ years). All patients were diagnosed as cStage II or III by pretreatment diagnostic imaging. All patients underwent R0 resection 6 to 10 weeks after CRT. According to the postoperative pathological classification defined by ypStage using resected specimens, 11 patients $(15.3 \%)$ were diagnosed as ypStage 0 (pCR), 20 as ypStage I, 30 as ypStage II, and 11 as ypStage III.

Sixty-four $(88.9 \%)$ patients completed all the process of CRT without severe adverse events. However, 8 patients required interruption or reduction of CRT. Clavien-Dindo IIIa or more postoperative complications were observed in 14 (19.4\%) patients. Among them, pelvic sepsis was the most frequently observed ( 5 patients) complication and ileus was the second most observed complication (4 patients). With regard to the administration of postoperative chemotherapy, 35 patients underwent chemotherapy. Among these 35 patients, 21 patients were administered a single FL-based chemotherapy, such as infusional 5-fluorouracil or an oral prodrug. The other 14 patients were treated with a combination of FL and OX. Eleven patients were diagnosed to be in the high NAR group and the others in the low NAR group.

Correlation between clinicopathological factors and diseaserecurrence. The overall incidence of disease recurrence was $27.8 \%$ (20/72). The correlations between various clinicopathological factors and disease recurrence are shown in Table II. In the univariate analysis, no significant correlations existed among gender, age, pretreatment clinical 
A

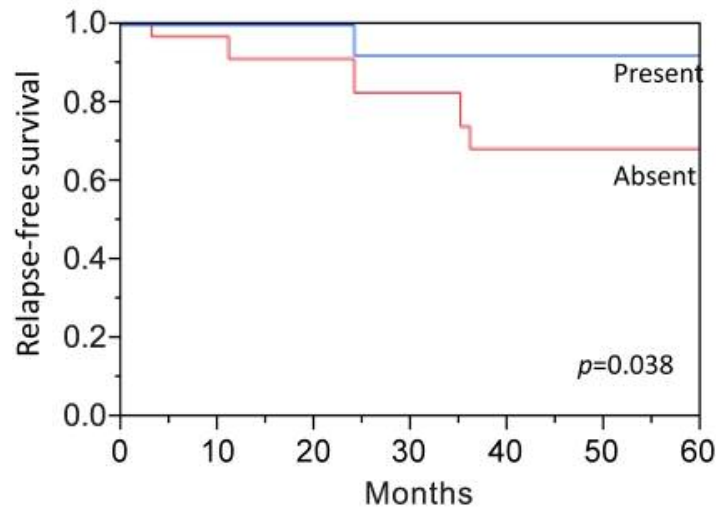

B

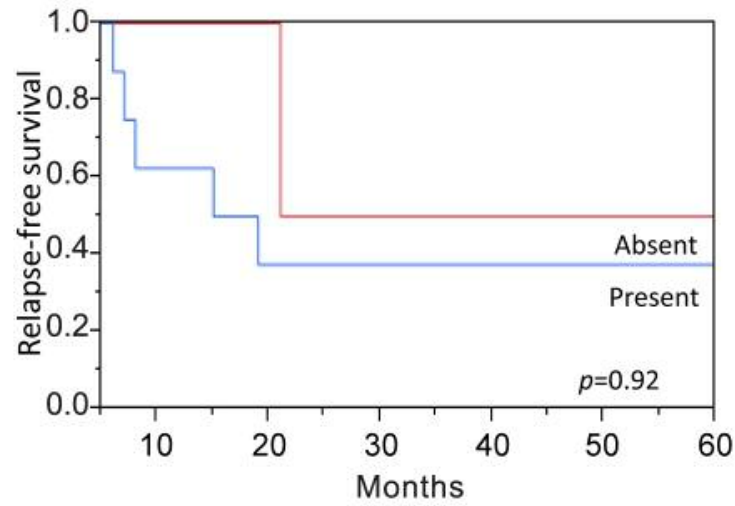

C

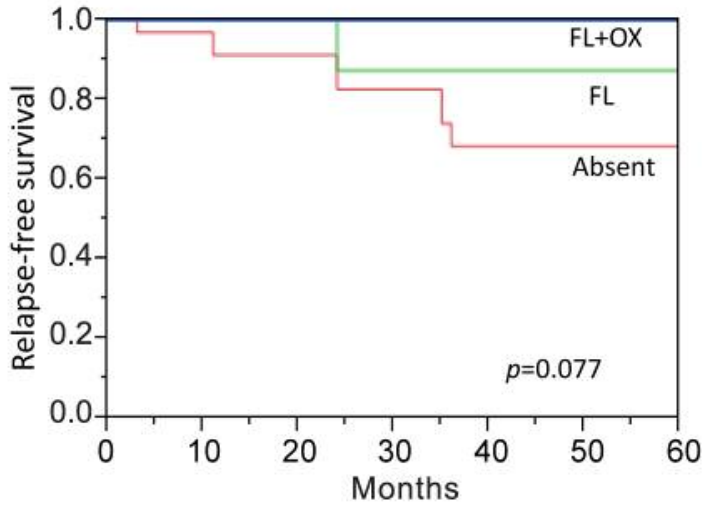

D

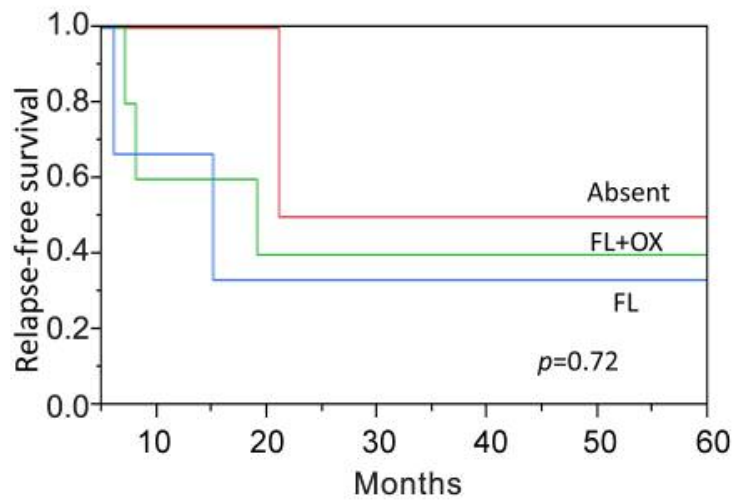

Figure 1. The relapse-free survival subdivided by a combination of NAR score and postoperative adjuvant chemotherapy. The prognosis was significantly $(p=0.038)$ better in the patients who received postoperative adjuvant therapy in the low NAR group (Figure 1A). Whereas, no difference existed between postoperative adjuvant therapy and survival in the high NAR group (Figure 1B). No correlation was observed between regimen and survival, based on the types of adjuvant chemotherapy regimen (Figure $1 C, D$ ).

stage, ypStage, postoperative complications, postoperative adjuvant chemotherapy and disease recurrence. However, disease recurrence was significantly more often observed in the patients with incompletion of neoadjuvant CRT, TRG 34, and high NAR score. Moreover, multivariate analysis revealed that NAR score was an independent predictor of disease recurrence along with TRG.

We examined relapse-free survival subdivided by a combination of NAR score and postoperative adjuvant chemotherapy. As a result, the Kaplan-Meier analysis and log-rank test demonstrated that the prognosis was significantly $(p=0.038)$ better in the patients who received postoperative adjuvant therapy in the low NAR group (Figure 1A). Whereas, no difference existed between postoperative adjuvant therapy and survival in the high NAR group (Figure 1B). No correlation was observed between regimen and survival, regarding the types of adjuvant chemotherapy regimen used (Figure 1C, D).

\section{Discussion}

Preoperative chemoradiotherapy decreases the risk of local recurrence and remains a standard treatment for patients with LARC. However, the benefit of subsequent adjuvant chemotherapy for patients who have undergone neoadjuvant CRT is uncertain and a topic of intense debate (7-15). A number of studies, including several randomized studies and meta-analyses, have attempted to address this issue, however, they have collectively produced mixed and inconclusive results.

To avoid wasted medical expenses and adverse events caused by adjuvant chemotherapy, it is important to predict patients who will have disease recurrence after the administration of neoadjuvant CRT and who will benefit from adjuvant chemotherapy.

According to the predictive markers for survival, pathological TRG has been proposed to stratify tumor 
response and is thought as one of the prognostic factors in rectal cancer patients after neoadjuvant CRT (19-22). In fact, it was discovered to be an independent predictor of disease recurrence in the present study. However, the role of TRG remains controversial in rectal cancer $(23,24)$. TRG in rectal cancer depends on several factors, such as the dose and schedule of radiotherapy, combination with chemotherapy, and time between medical treatment and surgery. Moreover, histopathological standardization is still lacking (25).

Whereas, George et al. (26) have reported that not only the absolute ypStage, but also the degree of tumor downstaging strongly associated with prognosis in rectal cancer patients after neoadjuvant CRT. The NAR score is calculated based on data supported by the Valentini nomogram for OS, using only the clinical $\mathrm{T}$ stage and pathological $\mathrm{T}$ and $\mathrm{N}$ stages, and is thought to be suitable for standardization of the degree of downstaging (16). Recently, Yothers et al. (27) showed that the NAR score had greater predictive ability than pCR for survival. Moreover, Sun et al. (28) have also reported that NAR score could help in predicting disease-free survival in rectal cancer patients after neoadjuvant CRT. The strength of the NAR score is the incorporation of both pre-and post-CRT variables to reflect the initial tumor extent and tumor response. Therefore, it is thought to reflect the treatment effect more accurately than absolute ypStage.

However, it remains controversial which patients will benefit from adjuvant chemotherapy after neoadjuvant CRT. Bregom et al. (28) and Sainato et al. (29) have reported that adjuvant chemotherapy improved neither overall survival nor disease-free survival in all rectal cancer patients after neoadjuvant CRT. However, Turner et al. (30) have reported that adjuvant chemotherapy may improve survival for rectal cancer with pCR. Collette et al. (31) have reported a survival benefit from adjuvant chemotherapy in patients with ypT02 tumors. In the present study, although no difference existed between postoperative adjuvant therapy and survival in the high NAR group, prognosis was significantly better in the patients who received postoperative adjuvant therapy in the low NAR group. From these results, it was suggested that adjuvant chemotherapy may improve survival of patients with a high therapeutic effect from neoadjuvant CRT.

In the present study, analysis of survival according to adjuvant chemotherapy revealed that no significant difference was observed in patients with single-FL based chemotherapy and patients with $\mathrm{FL}+\mathrm{OX}$ combination chemotherapy. However, Hong et al. (32) reported that adjuvant FOLFOX may improve disease-free survival compared with single FL-based adjuvant chemotherapy. A further large-scale prospective randomized trial is needed to evaluate the true effects of adjuvant therapy.

The present study has several limitations. First, this was a retrospective study with a small sample size of 72 patients. Second, selection bias may have been introduced in the decision to administer postoperative adjuvant therapy. A further large-scale prospective randomized trial is, therefore, needed to evaluate the true effects of adjuvant therapy.

In conclusion, NAR score may be one of the predictive markers for disease recurrence in patients who underwent neoadjuvant CRT followed by surgery for rectal cancer. The patients with low NAR score may benefit from postoperative adjuvant chemotherapy. This was a small retrospective study conducted at only two institutions and further larger multicenter studies are needed to clarify these findings.

\section{Conflicts of Interest}

The Authors have no conflicts of interest to declare regarding this study.

\section{Authors' Contributions}

Kiyoshi Maeda designed the study, analysed the data and wrote the draft of the manuscript. Masatsune Shibutani collected the clinical data and revised the manuscript critically; Akiko Tachimori, Takafumi Nishii, Naoki Aomatsu and Hisashi Nagahara helped collecting the updated clinical data of the patients. Tastunari Fukuoka performed the statistical analysis. Hiroshi Otani and Toru Inoue designed the study. Masaichi Ohira designed the study and critically reviewed the manuscript.

\section{Acknowledgements}

There is no financial support or relationship that may pose any conflict of interest.

\section{References}

1 Kapiteijin E, Maijnen CAM, Nagtegaal ID, Putter H, Steup WH, Wiggers T, Rutten H, Pahlman L, Glimelius B, van Krieken JHJM, Leer JWH and van de Velde CJH: Preoperative radiotherapy combined with total mesorectal excision for resectable rectal cancer. New Eng J Med 345: 638-646, 2001. PMID: 11547717. DOI: 10.1056/NEJMoa010580.

2 Colorectal Cancer Collaborative Group: Adjuvant radiotherapy for rectal cancer: a systematic overview of 8507 patients from 22 randomised trials. Lancet 358: 1291-1304, 2001. PMID: 11684209. DOI: 10.1016/S0140-6736(01)06409-1

3 Bosset JF, Collette L, Callais G, Mineur L, Maingon P, Radosevic-Jelic L, Daban A, Bardet E, and Beny A and Ollier JC: Chemotherapy with preoperative radiotherapy in rectal cancer. New Eng J Med 355: 1114-1123, 2006. PMID: 16971718. DOI: $10.1056 /$ NEJMoa060829

4 Saif MW, Hashmi S, Zelterman D, Almhanna K and Kim R: Capecitabine vs Contnuous infusion 5FU in neoadjuvant treatment of rectal cancer. A retrospective review. Int J Colorectal Dis 32: 139-145, 2008. PMID: 17909820. DOI: 10.1007/s00384-0070382-Z

5 Li Y, Wang J, Ma X, Tan L, Yan Y, Xue C, Hui B, Liu R, Hailin Ma and Ren J: A review of neoadjuvant chemoradiotherapy for locally advanced rectal cancer. Int J Biol Sci 12: 1022-1031, 2016. PMID: 27489505. DOI: 10.7150/ijbs.15438 
6 Rectal Cancer, NCCN Clinical Practice Guidelines in Oncology, Version2, 2019. Available at: http://www.nccn.org/professonal/ physician_gls/pdf/rectal.pdf (Last accessed on 3rd August 2019)

7 Bujko K, Glimelius B, Valentini V, Michalski W and Spalek M: Postoperative chemotherapy in patients with rectal cancer receiving preoperative radio(chemo)therapy: A meta-analysis of randomized trials comparing surgery \pm a fluoropyrimidine and surgery+a fluoropyrimidine \pm oxaliplatin. Eur J Surg Oncol 41: 713772, 2015. PMID: 25911110. DOI: 10.1016/j.ejso.2015.03.233

8 Petrelli F, Coinu A, Lonati V and Barni S: A systematic review and meta-analysis of adjuvant chemotherapy after neoadjuvant treatment and surgery for rectal cancer. Int J Colorectal Dis 30: 447-457, 2015. PMID: 25433820. DOI: 10.1007/s00384-0142082-9

9 Sauer R, Fietkau R, Wittekind C, Rodel C, Martus P, Hohenberger W, Tscmelitsch J, Sabitzer H, Karstens JH, Becker H, Hess C and Raab R: Adjuvant vs neoadjvant radiochemotherapy for locally advanced rectal cancer: the German trial CAO/ARO/AIO-94. Colorectal Dis 5: 406-415, 2003. PMID: 12925071.

10 Haynes AB, You YN, Hu CY, Eng C, Kopetz ES, RodriguezBigas MA, Skibber JM, Cantor SB and Chang GJ: Postoperative chemotherapy use after neoadjuvant chemoradiotherapy for rectal cancer: Analysis of Surveillance, Epidemiology, and End Results-Medicare data, 1998-2007. Cancer 120: 1162-1170, 2014. PMID: 24474245. DOI: $10.1002 /$ cncr.28545

11 Benson AB Rd: Should we consider adjuvant therapy for rectal cancer after neoadjuvant chemoradiotherapy? Clin Adv Hematol Oncol 14: 778-781, 2016. PMID: 27930628.

12 Heo SJ, Park HS, Kim JH, Choe EA, Koom WS, Hur H, Min BS, Kim NK, Kim H, Kim C, Jung I and Jung M: Role of adjuvant chemotherapy in locally advanced rectal cancer with ypT0-3N0 after preoperative chemoradiation therapy and surgery. BMC Cancer 17: 615-624, 2017. PMID: 28865435. DOI: $10.1186 / \mathrm{s} 12885-017-3624-7$

13 André T, Boni C, Navarro M, Tabernero J, Hickish T, Topham C, Bonetti A, Clingan P, Bridgewater J, Rivera F and de Gramont A: Improved overall survival with oxaliplatin, fluorouracil, and leucovorin as adjuvant treatment in stage II or III colon cancer in the MOSAIC trial. J Clin Oncol 7: 31093116, 2009. PMID: 19451431. DOI: 10.1200/JCO.2008.20.6771

14 Kiran RP, Kirat HT, Burgess AN, Nisar PJ, Kalady MF and Lavery IC: Is Adjuvant chemotherapy really needed after curative surgery for rectal cancer patients who are node-negative after neoadjuvant chemoradiotherapy? Ann Surg Oncol 19: 1206-1212, 2012. PMID: 21935748. DOI: 10.1245/s10434-0112044-1

15 Kahn KL, Adams JL, Weeks JC, Chrischilles EA, Schrag D, Ayanian JZ, Kiefe CI, Ganz PA, Bhoopalam N, Potosky AL, Harrington DP and Fletcher RH: Adjuvant chemotherapy use and adverse events among older patients with stage III colon cancer. JAMA 303: 1037-1045, 2010. PMID: 20233821. DOI: 10.1001/jama.2010.272

16 Valentini V, van Stiphout RG, Lammering G, Gambacorta MA, Barba MC, Bebenek M, Bonnetain F, Bosset JF, Bujko K, Cionini L, Gerard JP, Rödel C, Sainato A, Sauer R, Minsky BD, Collette L and Lambin P: Nomograms for predicting local recurrence, distant metastases, and overall survival for patients with locally advanced rectal cancer on the basis of European randomized clinical trials. J Clin Oncol 29: 3163-3172, 2011. PMID: 21747092. DOI: 10.1200/JCO.2010.33.1595
17 Clavien PA, Barkun J, de Oliveira ML, Vauthey JN, Dindo D, Sculik RD, de Santibanes E, Pekolj J, Slankamenac K, Bassi C, Graf R, Vonlanthen R, Padbury R, Cameron JL and Makuuchi M: The Clavien-Dindo classification of surgical complications: five-year experience. Ann Surg 250: 187-196, 2002. PMID: 19638912. DOI: 10.1097/SLA.0b013e3181b13ca2

18 Mandard AM, Dalibard F, Mandard JC, Marnay J, Henry-Amar M, Petiot JF, Roussel A, Jacob JH, Segol P, Samama G, Ollivier JM, Bonvalot S and Gignoux M: Pathologic assessment of tumor regression after preoperative chemoradiotherapy of esophageal carcinoma. Clinicopathologic correlations. Cancer 73: 26802686, 1994. PMID: 8194005. DOI: 10.1002/10970142(19940601)73:11<2680::aid-cncr2820731105>3.0.co;2-c

19 Fan WH, Xiao J, An X, Jiang W, Li LR, Gao YH, Chen G, Kong LH, Lin JZ, Wang JP, Pan ZZ and Ding PR: Patterns of recurrence in patients achieving pathologic complete response after neoadjuvant chemoradiotherapy for rectal cancer. J Cancer Res Clin Oncol 143: 1461-1467, 2017. PMID: 28386648. DOI: 10.1007/s00432-017-2383-9

20 De-Torres-Olombrada MV, Juez-Martel I, Rodriguez-Caravaca $\mathrm{G}$ and Duran-Poveda M: Role of the complete pathological response in rectal cancer: Value as a prognostic factor. J Surg Oncol, 2019. DOI: 10.31487/j.JSO.2018.01.005

$21 \mathrm{Hu}$ X, Li YQ, Ma XJ, Zhang L, S Cai SJ and PengJJ: Adjuvant chemotherapy for rectal cancer with complete pathological response (pCR) may not be necessary: a pooled analysis of 5491 patients. Cancer Cell Int 19: 127, 2019. DOI: 10.1186/s12935019-0851-9

22 Dossa F, Acuna SA, Rickles AS, Berho M, Wexner SD, Quereshy FA, Baxter NN and Chadi SA: Association between adjuvant chemotherapy and overall survival in patients with rectal cancer and pathological complete response after neoadjuvant chemotherapy and resection. JAMA Oncol 4: 930-937, 2017. PMID: 29710274. DOI: 10.1001/jamaoncol.2017.5597

23 Bonnetain F, Bosset JF, Gerard JP, Calais G, Conroy T, Mineur L, Bouché O, Maingon P, Chapet O, Radosevic-Jelic L, Methy $\mathrm{N}$ and Collette L: What is the clinical benefit of preoperative chemoradiotherapy with 5FU/leucovorin for T3-4 rectal cancer in a pooled analysis of EORTC 22921 and FFCD 9203 trials: surrogacy in question? Eur J Cancer 48: 1781-1790, 2012. PMID: 22507892. DOI: 10.1016/j.ejca.2012.03.016

24 Maas M, Nelemans PJ, Valentini V, Rödel C, Kuo LJ, Calvo FA, García-Aguilar J, Glynne-Jones R, Haustermans K, Mohiuddin M, Pucciarelli S, Small W Jr, Suárez J, Theodoropoulos G, Biondo S, Beets-Tan RG and Beets GL: Long-term outcome in patients with a pathological complete response after chemoradiation for rectal cancer: a pooled analysis of individual patient data. Lancet Oncol 11: 835-844, 2011. PMID: 20692872. DOI: $10.1016 / \mathrm{S} 1470-2045(10) 70172-8$

25 Chetty R, Gill P, Adrian C Bateman, Driman DK, Govender D, Bateman AR, Chua YJ, Greywoode G, Hemmings C, Imat I, JaynesE, Lee CS, Locketz M, Rowsell C, Rullier A, Serra S, Szentgyorgyi E, Vajpeyi R, Delaney D and Wang LM: Pathological grading of regression: an international Study Group perspective. J Clin Pathol 65: 865-866, 2012. PMID: 22859397. DOI: 10.1136/jclinpath-2012-201054

26 George TJ, Allegra CJ and Yothers G: Neoadjuvant rectal (NAR) score: new surrogate endopoint in rectal cancer clinical trials. Curr Colorectal Cancer Rep 11: 275-280, 2015. PMID: 26321890. DOI: $10.1007 / \mathrm{s} 11888-015-0285-2$ 
27 Yothers G, George TJ, Allegra CJ, Bosset JF, Bujko K, Collette L, O'Connell MJ, Doyen J, Fernandez-Martos C, Seitz JF and Wolmark N: Predictive validity of neoadjuvant rectal (NAR) score and pathologic complete response (ypCR) for overall survival (OS) as surrogate endpoints in rectal cancer clinical trial. J Clin Oncol 34(Suppl 15): 3533, 2017. DOI: 10.1200/JCO.2016.34.15_suppl.3533

28 Breugom AJ, Swets M, Bosset JF, Collette L, Sainato A, Cionini L, Glynne-Jones R, Counsell N, Bastiaannet E, van de Broek CB, Liefers GJ, Putter $\mathrm{H}$ and van de Velde CJ: Adjuvant chemotherapy after preoperative (chemo)radiotherapy and surgery for patients with rectal cancer: a systematic review and meta-analysis of individual patient data. Lancet Oncol 16: 200207, 2015. PMID: 25589192. DOI: 10.1016/S1470-2045 (14)71199-4

29 Sainato A, Cernusco Luna Nunzia V, Valentini V, De Paoli A, Maurizi ER, Lupattelli M, Aristei, Cristiana C, Vidali C, Conti M, Galardi A, Ponticelli P, Friso ML, Iannone T, Osti FM, Manfredi B, Coppola M, Orlandini C and Cionini L: No benefit of adjuvant Fluorouracil Leucovorin chemotherapy after neoadjuvant chemoradiotherapy in locally advanced cancer of the rectum (LARC): Long term results of a randomized trial (ICNR-RT). Radiother Oncol 113: 223-229, 2014. PMID: 25454175. DOI: $10.1016 /$ j.radonc.2014.10.006

30 Turner MC, Keenan JE, Rushing CN, Gulack BC, Nussbaum DP, Benrashid E, Hyslop T, Strickler JH, Mantyh CR and Migaly $\mathrm{J}$ : Adjuvant chemotherapy improves survival following resection of locally advanced rectal cancer with pathologic complete response. J Gastrointest Surg 23: 1614-1622, 2019. PMID: 30635829. DOI: $10.1007 / \mathrm{s} 11605-018-04079-8$
31 Collette L, Bosset JF, den Dulk M, Nguyen F, Mineur L, Maingon P, Radosevic-Jelic L, Piérart M and Calais G: European Organisation for Research and Treatment of Cancer Radiation Oncology Group. Patients with curative resection of cT3-4 rectal cancer after preoperative radiotherapy or radiochemotherapy: does anybody benefit from adjuvant fluorouracil-based chemotherapy? A trial of the European Organisation for Research and Treatment of Cancer Radiation Oncology Group. J Clin Oncol 25: 4379-4386, 2007. PMID: 17906203. DOI: 10.1200/JCO.2007.11.9685

32 Hong YS, Nam BH, Kim KP, Kim JE, Park SJ, Park JO, Kim SY, Kim TY, Kim JH, Ahn JB, Lim SB, Yu CS, Kim JC, Yun SH, Kim JH, Park JH, Jung KH and Kim TW: Oxaliplatin, fluorouracil, and leucovorin versus fluorouracil and leucovorin as adjuvant chemotherapy for locally advanced rectal cancer after preoperative chemoradiotherapy (ADORE): an open-label, multicentre, phase 2, randomised controlled trial. Lancet Oncol 15: 1245-1253, 2014. PMID: 25201358. DOI: 10.1016/S14702045(14)70377
Received September 7, 2019

Revised October 16, 2019

Accepted October 17, 2019 